\section{Guillemin, Roger Charles Louis}

W. Hubl

Ehem. Krankenhaus Dresden-Friedrichstadt, Institut für

Klinische Chemie und Labormedizin, Dresden, Deutschland

Lebensdaten Französischer Neuroendokrinologe, geboren am 11. Januar 1924 in Dijon, Côte-d'Or (Frankreich). Guillemin schloss an der Universität Dijon mit dem B. A. und dem B. Sc. ab. Er arbeitete bei Hans Selye in Montreal über die Desoxykortikosteron-induzierte Hypertonie und promovierte im Jahr 1949 zum Doktor der Medizin an der Universität in Lyon. 1953 erhielt er den Ph. D. von der Universität in Montreal. Von 1953-1970 lehrte er Physiologie an der Baylor Universität in Houston, Texas. Im Jahr 1970 etablierte er im Salk-Institute, in La Jolla, Kalifornien, ein Labor für Neuroendokrinologie. Dort isolierte er den ersten hypothalamischen ReleasingFaktor Thyreotropin-Releasing-Faktor TRF ( $\triangleright$ ThyreotropinReleasing-Hormon), und in den darauf folgenden Jahren den Luteotropic-Hormon-Releasing-Faktor ( $\vee$ GonadotropinReleasing-Hormon). Er produzierte zahlreiche synthetische Analoge der Hypothalamushormone, z. B. von $>$ Somatostatin sowie GnRH, und isolierte Endorphine. Im Jahr 1989 trat Guil- lemin in den Ruhestand und stellte seine jahrelangen Erfahrungen für die Entwicklung von Computerprogrammen zur Überführung von wissenschaftlichen Ergebnissen in die Kunst zur Verfügung. Als Interims-Präsident des Salk-Instituts organisierte er von 2007-2009 Kunstausstellungen zur Verbindung der Wissenschaft mit der Kunst.

Verdienste Im Jahr 1974 wurde er zum Mitglied der National Academy of Science der USA gewählt und 2 Jahre später zum Mitglied der American Academy of Arts and Science. Er erhielt zahlreiche Preise, beispielsweise 1975 den Lasker Award in Basic Sciences, 1976 den Gairdner International Award, Toronto. Im Jahr 1977 erhielt er zusammen mit Andrew Victor Schally den Nobelpreis für Physiologie und Medizin ,für die Entdeckungen über die Produktion von Peptidhormonen im Gehirn“.

\section{Literatur}

Lindsten J (1992) Nobel Lectures in physiology or medicine 1971-1980. Karolinska Institute, Stockholm

Quincy FM (2011) Roger Guillemin. Gonadotropin-releasing hormone. Stapress-Verlag, Beau Bassin-Rose Hill, Mauritius 